\title{
ARITHMETICS OF RATIONAL GENERALIZED QUATERNION ALGEBRAS
}

\author{
D. M. BROWN
}

1. Introduction. A rational generalized quaternion algebra is a linear associative algebra of order four and rank two, having a principal modulus $I_{0}$, ranging over the field of rational numbers. A basis $\left(I_{0}, I_{1}, I_{2}, I_{1} I_{2}\right)$ may be found ${ }^{1}$ such that

(1) $I_{1}^{2}=\alpha, \quad I_{2}^{2}=\beta, \quad I_{1} I_{2}=-I_{2} I_{1}$, $\alpha$ and $\beta$ rational integers.

An algebra in such form will be denoted by $Q(\alpha, \beta)$, a number in it by

$$
Q=a_{0} I_{0}+a_{1} I_{1}+a_{2} I_{2}+a_{12} I_{1} I_{2}, \quad a \text { 's rational, }
$$

and its norm by

$$
N(Q)=a_{0}^{2}-\alpha a_{1}^{2}-\beta a_{2}^{2}+\alpha \beta a_{12}^{2} .
$$

$Q$ satisfies its rank equation

$$
X^{2}-2 a_{0} X+N(Q)=0 .
$$

$a_{0}$ is called the real part of $Q$.

An arithmetic $S$ of $Q(\alpha, \beta)$ is a set of numbers having the following properties:

$\mathrm{C}_{\mathrm{a}}: S$ is closed with respect to algebraic addition.

$\mathrm{C}_{\mathrm{m}}: S$ is closed with respect to multiplication.

$\mathrm{R}$ : For every number of $S$, (4) has integral coefficients.

$\mathrm{U}: S$ contains $I_{0}, I_{1}$ and $I_{2}$ (and hence $I_{1} I_{2}$ by $\mathrm{C}_{\mathrm{m}}$ ).

$\mathrm{M}: S$ is maximal; that is, $S$ is contained in no larger set having Properties $\mathrm{C}_{\mathrm{a}}, \mathrm{C}_{\mathrm{m}}, \mathrm{R}$ and $\mathrm{U}$.

It is the purpose of this paper to determine a set of bases for the arithmetics of those algebras for which $\alpha$ and $\beta$ contain no squared prime factors. ${ }^{2}$ In any case, it has been proved $^{3}$ that for a given arith-

${ }^{1}$ Dickson, L. E., Algebren und ihre Zahlentheorie, Zurich, 1927, pp. 43-44. The definition of an arithmetic is made there also.

${ }^{2}$ If $\alpha$ and $\beta$ contain squared prime factors, the number of arithmetics varies with the form of those factors. See M. Eichler, Untersuchungen in der Zahlentheorie der rationalen Quaternionalgebren, Journal für die reine und angewandte Mathematik, vol. 174 (1936), p. 149, Theorem 12.

${ }^{3}$ Latimer, C. G., The classes of integral sets in a quaternion algebra, Duke Mathematical Journal, vol. 3 (1937), pp. 246-247, \$7. On pages $237-238$, \$2, of this reference is stated a theorem giving necessary and sufficient conditions that a basis of $Q(\alpha, \beta)$ be a basis of an arithmetic of $Q\left(\alpha^{\prime}, \beta^{\prime}\right)$. 
metic $S$ in the algebra, a basis $\left(I_{0}^{\prime}, I_{1}^{\prime}, I_{2}^{\prime}, I_{1}^{\prime} I_{2}^{\prime}\right)$ can be found such that $I_{1}^{\prime 2}=\alpha^{\prime}, I_{2}^{\prime 2}=\beta^{\prime}, I_{1}^{\prime} I_{2}^{\prime}=-I_{2}^{\prime} I_{1}^{\prime}, \alpha^{\prime}$ and $\beta^{\prime}$ containing no squared prime factors, and such that the given arithmetic is an arithmetic of $Q\left(\alpha^{\prime}, \beta^{\prime}\right)$. The problem of determining bases for the arithmetics of such algebras has been solved by Latimer ${ }^{4}$ when $\alpha \equiv \beta \equiv 1(\bmod 2)$, and by Darkow ${ }^{5}$ when $\alpha \equiv \beta \equiv 0(\bmod 2)$. Darkow's results can be extended to apply to the remaining cases $($ when $\alpha+\beta \equiv 1(\bmod 2)) .{ }^{6}$ All possible cases are treated in this paper, with greatly simplified results. $^{7}$

Albert ${ }^{8}$ has shown that every rational generalized quaternion $d i$ vision algebra $Q(\alpha, \beta)$ may be transformed into $Q(\tau, \sigma)$, in which $\tau$ and $\sigma$ have special properties. Concerning the treatments by Latimer and Darkow (similar to that used in this paper) Albert writes: ${ }^{9}$

The.. division into special cases is certainly not desirable. Nor is it nezessary. For it is obvious that at least an attempt should be made to show that transformations carrying all cases into a canonical form are possible, and it is this canonical form which should be studied. . . In particular, it is evident that the integral sets of $S$ should contain the integers of the realm $R(i)$.

Concerning the above quotation, a few comments seem pertinent. Albert's results are readily obtainable from the results of this paper or those of Latimer and Darkow. His canonical form is not unique, and the number (two) of arithmetics obtained by it is, in general, much less than the number of arithmetics as defined by this paper. There being no essential difference between the basal elements $I_{1}$ and $I_{2}$, or, for that matter, $I_{1} I_{2}$, there seems to be little reason for requiring that the integers of the realm $R\left(I_{1}\right)$ be contained in an integral set (arithmetic) in preference to those of realms $R\left(I_{2}\right)$ or $R\left(I_{1} I_{2}\right)$. Albert's results apply only to division algebras, while those of Latimer and Darkow and the writer apply to all algebras. The norm of a number of $Q(\alpha, \beta)$, given in (3), being of a form of great interest in number theory, it would seem particularly useful to have

\footnotetext{
${ }^{4}$ Latimer, C. G., Arithmetics of generalized quaternion algebras, American Journal of Mathematics, vol. 48 (1926), pp. 57-66.

${ }^{5}$ Darkow, M. D., Determination of a basis for the integral elements of certain generalized quaternion algebras, Annals of Mathematics, (2), vol. 28 (1926), pp. 263-270.

${ }^{6}$ Latimer, C. G., On the class number of a quaternion algebra with a negative fundamental number, Transactions of this Society, vol. 40 (1936), p. 320.

7 The writer is greatly indebted to Dr. Claiborne G. Latimer for valuable suggestions enabling much greater simplification than he had previously obtained.

${ }^{8}$ Albert, A. A., Integral domains of rational generalized quaternion algebras, this Bulletin, vol. 40 (1934), pp. 164-176.

${ }^{9}$ Albert, A. A., loc. cit., p. 165. The $i$ used there is the same as the $I$ used in this paper.
} 
available a method of obtaining the arithmetics of an algebra expressed in terms of parameters $\alpha$ and $\beta$ which yield that exact norm. Clearly Albert's canonical form would not suffice, so that methods of treatment as given here are conceivably not only useful but necessary.

As mentioned above, the method of treatment in this paper is similar to that used by Latimer and Darkow, the work being more direct in some places. ${ }^{10}$

2. Notations, transformations and definitions used. The letters $r, s$ and $t$ will be used exclusively to denote permutations of 1,2 , and 3 . Let $(g-1)(g+1)=0$ such that

$$
g=\left\{\begin{array}{l}
1 \text { if the number of inversions of }(r s t) \text { is even, } \\
-1 \text { otherwise. }
\end{array}\right.
$$

Let the g.c.d. of $\alpha$ and $\beta$ be

$$
(\alpha, \beta)=\gamma_{3} \text { (positive or negative) }
$$

and $\alpha=-\gamma_{2} \gamma_{3}, \beta=\gamma_{3} \gamma_{1}$. Let

$$
I_{1} I_{2}=\gamma_{3} I_{3}
$$

so that from (1), $I_{r}^{2}=-\gamma_{s} \gamma_{t}$, and $I_{r} I_{s}=g \gamma_{t} I_{t}$. From the results of Latimer and Darkow, it can easily be shown that $I_{3}$ is in each arithmetic, as are $I_{1}$ and $I_{2}$. Hence from (6) and (7), $I_{1}, I_{2}$, and $I_{3}$ are formally identical, as are the $\gamma$ 's, the latter being formally identical to their negatives. $Q(\alpha, \beta)$ will hereafter be written in the symmetric notation

$$
Q\left(\gamma_{1}, \gamma_{2}, \gamma_{3}\right) \equiv Q(\alpha, \beta)
$$

Making the unitary transformation

$$
\begin{gathered}
i_{0}=g I_{0}, \quad i_{1}=g I_{r}, \quad i_{2}=g I_{s}, \quad i_{3}=g I_{t}, \\
\gamma_{r}=\alpha_{1}, \quad \gamma_{s}=\alpha_{2}, \quad \gamma_{t}=\alpha_{3},
\end{gathered}
$$

one has $i_{r}^{2}=-\alpha_{s} \alpha_{t}$ and $i_{r} i_{s}=g \alpha_{t} i_{t}$, which is abstractly identical with (9). $Q\left(\gamma_{1}, \gamma_{2}, \gamma_{3}\right)$ now becomes $Q\left(\alpha_{1}, \alpha_{2}, \alpha_{3}\right)$. After proper change of sign of the $\gamma^{\prime}$ 's, one finds that $\gamma_{t}$ is odd or even, $\gamma_{s} \equiv 1(\bmod 4), \gamma_{r}$ is odd, and $\gamma_{r} \equiv 1(\bmod 4)$ if $\gamma_{t}$ is odd. Choosing

$$
(\theta-1)(\theta-2)=0 \text {, }
$$

$Q\left(\alpha_{1}, \alpha_{2}, \alpha_{3}\right)$ is such that

${ }^{10}$ Latimer and Darkow obtain their results for $Q(\alpha,-\beta)$. Each mentions a "tentative" process for obtaining bases. See Latimer, loc. cit., footnote 3, pp. 61 and 65; Darkow, loc. cit., p. 263 and p. $267 \mathrm{ff}$. 


$$
\alpha_{3} \equiv \theta(\bmod 2 \theta), \quad \alpha_{2} \equiv 1(\bmod 4), \quad \alpha_{1} \equiv \alpha_{2}(\bmod 2(3-\theta)) .
$$

In what follows, it will be assumed that (9) has been made so that (11) holds. All other symbols introduced hereafter will represent integers, unless they are obviously not integers. The parameters $\theta, m, k$, $n, A_{r}, B_{r}, \xi$ and $H_{j}$, used frequently hereafter, and most easily determined in the order given, are defined by the following, each being unique save $n$ and $H_{j}$ :

$\theta: \alpha_{3} \equiv \theta(\bmod 2 \theta) ;(\theta-1)(\theta-2)=0$.

$m:\left(\alpha_{1}+\alpha_{2}+m \alpha_{3}\right) \equiv 2-\theta(\bmod 4 \theta) ;(m-1)(m+1)[(\theta-1) m(m+2)$ $+2-\theta]=0$.

$k: 2\left(m^{2}+m+1\right) k=(m+1)(m+2) .^{11}$

$n: k n^{2}-n=0$.

$A_{r}, B_{r}: \alpha_{r}=A_{r} B_{r}$, and

$$
\left(\frac{-\alpha_{s} \alpha_{t}}{A_{r}}\right)=1, \quad\left(\frac{-\alpha_{s} \alpha_{t}}{B_{r}}\right)=-1,
$$

$B_{r}$ positive and a minimum. ${ }^{12}$

$\xi: \xi$ is the number of prime factors (all distinct) of $A_{1} A_{2} A_{3}$.

$H_{j}:\left(\alpha_{1} \alpha_{2}+\alpha_{2} \alpha_{3}+\alpha_{3} \alpha_{1}\right) H_{j}^{2} \equiv-1\left(\bmod A_{1} A_{2} A_{3}\right),(j=1,2, \cdots, \xi){ }_{.13}$ If $H_{j}$ is even, then $H_{j}^{\prime}=H_{j} \pm A_{1} A_{2} A_{3}$ is odd. Let $f_{7}=(1-2 m)$ and $f_{8}=2 \theta k-2 k+2$. Then if $A_{2} B_{1} H_{j}^{\prime} \not \equiv f_{7}\left(\bmod f_{8}\right)$, it can be shown that $H_{j}^{\prime \prime}=H_{j}^{\prime} \pm A_{1} A_{2} A_{3}$ is such that $A_{2} B_{1} H_{j}^{\prime \prime} \equiv f_{7}\left(\bmod f_{8}\right)$. Since $H_{j}, H_{j}^{\prime}$, and $H_{j}^{\prime \prime}$ are all in the same residue class, modulo $A_{1} A_{2} A_{3}$, it follows (and will be so assumed hereafter) that $H_{j}$ may always be chosen such that $\left(f_{7}\right.$ is odd and $f_{8}$ is even, so $H_{j}$ will be odd)

$$
A_{2} B_{1} H_{j} \equiv f_{7}\left(\bmod f_{8}\right) \text {. }
$$

The following symbols will also be used:

$$
\begin{aligned}
& f_{1}=(2-\theta) k n, f_{2}=(\theta n-2 n+2), f_{3}=k(m+\theta n-2 n), \\
& f_{4}=(2-\theta)(m-2 n-k+1)+n, f_{5}=(2 n-\theta n-k+2), \\
& f_{6}=(\theta-1)(m-2 k+2), f_{7}=(1-2 m), f_{8}=2 \theta k-2 k+2,
\end{aligned}
$$

11 If $e=1-k$, then $k$ may also be defined by $\left(\alpha_{1}+\alpha_{2}+\alpha_{3}\right)\left(\alpha_{1}+\alpha_{2}\right)\left(\alpha_{2}+\alpha_{3}\right)\left(\alpha_{3}+\alpha_{1}\right)$ $\equiv 8 e(\bmod 16)$. See Latimer, C. G., On the fundamental number of a rational generalized quaternion algebra, Duke Mathematical Journal, vol. 1 (1935), p. 435, theorem and second footnote.

12 In these conditions

$$
\left(\frac{A}{P}\right)
$$

is the Legendre symbol for quadratic residues.

${ }^{13}$ This congruence has exactly $\xi$ distinct solutions. See Dickson, L. E., Introduction to the Theory of Numbers, University of Chicago Press, 1929, p. 12, Theorem 16. 


$$
M_{j}=\frac{\alpha_{1} H_{j} i_{1}+i_{2}}{2 A_{3}}, \quad N_{j}=\frac{1}{2} i_{0}+\frac{-\alpha_{1} H_{j} i_{1}+\alpha_{2} H_{j} i_{2}+i_{3}}{2 A_{1} A_{2}} .
$$

3. The fundamental form of a number $Q$ in an arithmetic. From the relations given by (2), (3), (7) and (9), a number in $Q\left(\alpha_{1}, \alpha_{2}, \alpha_{3}\right)$ is such that

$$
\begin{aligned}
Q & =b_{0} i_{0}+b_{1} i_{1}+b_{2} i_{2}+b_{3} i_{3}, \\
N(Q) & =b_{0}^{2}+\alpha_{2} \alpha_{3} b_{1}^{2}+\alpha_{3} \alpha_{1} b_{2}^{2}+\alpha_{1} \alpha_{2} b_{3}^{2}
\end{aligned}
$$

(the $b$ 's rational). By Property $\mathrm{C}_{\mathrm{m}}$, if $Q$ is in $S$, then so are $Q i_{0}, Q i_{1}, Q i_{2}$ and $Q i_{3}$, whose real parts are $b_{0},-\alpha_{2} \alpha_{3} b_{1},-\alpha_{3} \alpha_{1} b_{2}$ and $-\alpha_{1} \alpha_{2} b_{3}$, respectively, and by Property $\mathrm{R}$ and (4), one may write $2 b_{0}=X_{0}$, $2 b_{r} \alpha_{s} \alpha_{t}=X_{r}$, so that (15) becomes

$$
\begin{aligned}
Q & =\frac{1}{2}\left[X_{0} i_{0}+\frac{X_{1} i_{1}}{\alpha_{2} \alpha_{3}}+\frac{X_{2} i_{2}}{\alpha_{3} \alpha_{1}}+\frac{X_{3} i_{3}}{\alpha_{1} \alpha_{2}}\right], \\
N(Q) & =\frac{1}{4}\left[X_{0}^{2}+\frac{X_{1}^{2}}{\alpha_{2} \alpha_{3}}+\frac{X_{2}^{2}}{\alpha_{3} \alpha_{1}}+\frac{X_{3}^{2}}{\alpha_{1} \alpha_{2}}\right]
\end{aligned}
$$

and, by Property R,

$$
\alpha_{1} \alpha_{2} \alpha_{3} X_{0}^{2}+\alpha_{1} X_{1}^{2}+\alpha_{2} X_{2}^{2}+\alpha_{3} X_{3}^{2} \equiv 0\left(\bmod 4 \alpha_{1} \alpha_{2} \alpha_{3}\right) .
$$

Using (11), (17) becomes equivalent to

$$
\begin{aligned}
\alpha_{1} \alpha_{2} \alpha_{3} X_{0}^{2}+\alpha_{1} X_{1}^{2}+\alpha_{2} X_{2}^{2}+\alpha_{3} X_{3}^{2} & \equiv 0(\bmod 4 \theta) \\
\text { if also } \alpha_{r} X_{r}^{2}+\alpha_{s} X_{s}^{2} & \equiv 0\left(\bmod \alpha_{t}\right) .
\end{aligned}
$$

Let $\delta_{t}$ be any prime factor of $\alpha_{t}$. If $\delta_{t}$ divides $X_{r}$, it also divides $X_{s}$. But if $\left(X_{r}, \delta_{t}\right)=1$, then there exists an integer $p_{r}$ such that $p_{r} X_{r} \equiv 1$ $\left(\bmod \delta_{t}\right)$. Hence from $(18), p_{r}^{2} \alpha_{r} X_{r}^{2}+p_{r}^{2} \alpha_{s} X_{s}^{2} \equiv 0\left(\bmod \delta_{t}\right), \alpha_{r}+p_{r}^{2} \alpha_{s} X_{s}^{2}$ $\equiv 0\left(\bmod \delta_{t}\right)$, or $\left(p_{r} \alpha_{s} X_{s}\right)^{2} \equiv-\alpha_{r} \alpha_{s}\left(\bmod \delta_{t}\right)$. Hence

$$
\left(\frac{-\alpha_{r} \alpha_{s}}{\delta_{t}}\right)=1
$$

and from (13), $X_{r}$ may be prime to each factor of $A_{t}$, but must be divisible by each factor of $B_{t}$, and hence by $B_{t}$ itself. Therefore $B_{t}$ divides $X_{r}$ and $X_{s}$, and one may write

$$
X_{0}=x_{0}, \quad X_{r}=B_{s} B_{t} x_{r},
$$

and (16) and (18) become, respectively, 


$$
\begin{aligned}
Q_{x} & =\frac{1}{2}\left[x_{0} i_{0}+\frac{x_{1} i_{1}}{A_{2} A_{3}}+\frac{x_{2} i_{2}}{A_{3} A_{1}}+\frac{x_{3} i_{3}}{A_{1} A_{2}}\right], \\
N(Q) & =\frac{1}{4}\left[x_{0}^{2}+\frac{B_{2} B_{3} x_{1}^{2}}{A_{2} A_{3}}+\frac{B_{3} B_{1} x_{2}^{2}}{A_{3} A_{1}}+\frac{B_{1} B_{2} x_{3}^{2}}{A_{1} A_{2}}\right],
\end{aligned}
$$

and

$$
\begin{gathered}
\alpha_{1} \alpha_{2} \alpha_{3} x_{0}^{2}+\alpha_{1} x_{1}^{2}+\alpha_{2} x_{2}^{2}+\alpha_{3} x_{3}^{2} \equiv 0(\bmod 4 \theta), \\
\left(A_{s} B_{r} x_{s}\right)^{2} \equiv-\alpha_{r} \alpha_{s} x_{r}^{2}\left(\bmod A_{t}\right) .
\end{gathered}
$$

Hereafter, the numbers $x_{0}, x_{1}, x_{2}, x_{3}$ will be referred to as the coordinates of a number $Q_{x}$ in $S$, and (20) will be called the fundamental form of $Q$ in $S$. Using the definition of $H_{j}$ to obtain $\alpha_{r} \alpha_{s} H_{j}^{2} \equiv-1$ $\left(\bmod A_{t}\right)$, and multiplying both members of $(21 \mathrm{~b})$ by $H_{j}^{2}$, one obtains $\left(A_{s} B_{r} H_{j} x_{s}\right)^{2} \equiv-\alpha_{r} \alpha_{s} H_{j}^{2} x_{r}^{2} \equiv x_{r}^{2}\left(\bmod A_{t}\right)$, or, reducing to linear congruences, $x_{r} \equiv A_{s} B_{r}\left( \pm H_{j}\right) x_{s}\left(\bmod A_{t}\right)$. Now if $H_{j}$ is a solution of its congruence, then $-H_{j}$ is another, distinct from $H_{j}$, and one may finally write

$$
x_{r} \equiv g A_{s} B_{r} H_{j} x_{s}\left(\bmod A_{t}\right),
$$

where $g$ is defined as in (5). Hence from (20), (21) and (22) one concludes:

THEOREM I. If a number $Q$ is in an arithmetic, it is necessary that it have the form (20), whose coordinates satisfy (21a) and (22).

4. Analysis of the conditions imposed on the coordinates of a number $Q$ of fundamental form lying in an arithmetic. It can be shown that two numbers of fundamental form have Property $\mathrm{C}_{\mathrm{a}}$ if and only if their coordinates satisfy (22) for the same value of $j$. Hereafter $H_{j}$ will be replaced by $H$ whenever the value of $j$ is fixed in an argument. Let $Q_{x}$ and $Q_{y}$ be in an arithmetic, and hence satisfy (22) for the same value of $j$. Then by Property $\mathrm{C}_{\mathrm{m}}, Q_{x} Q_{y}$ must also lie in $S$. A study of the coordinates of this new number reveals that in addition to (21a) and (22), the following conditions must hold: $:^{14}$

$$
\begin{aligned}
\alpha_{1} \alpha_{2} \alpha_{3} x_{0} y_{0}+\alpha_{1} x_{1} y_{1}+\alpha_{2} x_{2} y_{2}+\alpha_{3} x_{3} y_{3} & \equiv 0(\bmod 2 \theta), \\
x_{0} y_{1}+y_{0} x_{1}+y_{2} x_{3}+x_{2} y_{3} & \equiv 0(\bmod 2), \\
x_{0} y_{2}+y_{0} x_{2}+y_{3} x_{1}+x_{3} y_{1} & \equiv 0(\bmod 2), \\
\theta\left(x_{0} y_{3}+y_{0} x_{3}\right)+y_{1} x_{2}-x_{1} y_{2} & \equiv 0(\bmod 2 \theta) .
\end{aligned}
$$

${ }^{14}$ Here begins the direct method, as opposed to the "tentative" process of Latimer and Darkow. See footnote 10. 
Exhaustive analysis of (21a), (22) and (23) further reveals that the conditions that must hold in order for $Q_{x}$ and $Q_{y}$ to have property $M$ are as follows:

$$
\begin{aligned}
x_{0}= & {[(2-\theta) n+(\theta-1) k m] x_{1} } \\
& +(2-\theta)(1-n) x_{2}+(\theta-1) x_{3}(\bmod 2), \\
(24) \quad x_{1} \equiv & 0(\bmod \theta+k-\theta k), \\
x_{1} \equiv & (1-2 n) x_{2}+2 m x_{0}(\bmod \theta k+2 \theta-2 k), \text { for fixed } n, \\
x_{3} \equiv & (2-\theta)\left[(1-n) x_{1}+n x_{2}\right](\bmod 3-\theta) \\
(25) \quad x_{1} \equiv & B_{1} H\left(A_{2} x_{2}-A_{3} x_{3}\right)\left(\bmod A_{2} A_{3}\right), \quad x_{2} \equiv A_{3} B_{2} H x_{3}\left(\bmod A_{1}\right),
\end{aligned}
$$

which is equivalent to (22). Letting $P_{0}, P_{1}, P_{2}$, and $P_{3}$ be arbitrary integers, the $f^{\prime}$ 's integers as defined in (13), and $M_{j}$ and $N_{j}$ as in (14), then (24) and (25) can be reduced to

$$
\begin{array}{ll}
x_{0}=2 P_{0}+f_{1} P_{1}+f_{3} P_{2}+P_{3}, & \\
x_{1}=f_{2} A_{2} A_{3} P_{1}+\left(f_{4} A_{2} A_{3}+f_{5} A_{2} \alpha_{1} H\right) P_{2}+\left(f_{6} A_{2} A_{3}-A_{3} B_{1} H\right) P_{3}, & \\
x_{2}=f_{5} A_{1} P_{2}+A_{3} B_{2} H P_{3}, & \text { for fixed } n . \\
x_{3}=P_{3}, &
\end{array}
$$

Substituting these in (20a) one has

$$
\begin{aligned}
Q_{x j n}= & P_{0} i_{0}+\frac{1}{2}\left(f_{1} i_{0}+f_{2} i_{1}\right) P_{1}+\left[\frac{1}{2}\left(f_{3} i_{0}+f_{4} i_{1}\right)+f_{5} M_{j}\right] P_{2} \\
& +\left[\frac{1}{2} f_{6} i_{1}+N_{j}\right] P_{3}, \quad j=1,2, \cdots, \xi .
\end{aligned}
$$

Now let

$$
\begin{array}{ll}
U_{0 j n}=i_{0}, & U_{1 j n}=\frac{1}{2}\left(f_{1} i_{0}+f_{2} i_{1}\right), \\
U_{2 j n}=\frac{1}{2}\left(f_{3} i_{0}+f_{4} i_{1}\right)+f_{5} M_{j}, & U_{3 j n}=\frac{1}{2} f_{6} i_{1}+N_{j} .
\end{array}
$$

(27) then becomes

$$
Q_{x j n}=P_{0} U_{0}+P_{1} U_{1}+P_{2} U_{2}+P_{3} U_{3} .
$$

All such numbers, for a fixed $j$ and $n$, form an integral set of numbers with (28) as a basis. Since (28) is equivalent to (26) and (20) combined, and since (26) and (20) establish necessary and sufficient conditions that Properties $\mathrm{C}_{\mathrm{a}}, \mathrm{C}_{\mathrm{m}}, \mathrm{R}$ and $\mathrm{M}$ hold, and it is easily shown that $i_{1}, i_{2}$ and $i_{3}$ are of the form (29), so that Property $U$ holds, it follows that (28) gives the bases of the algebra-one for each value of $j$ and $n$. But $n$ has $k+1$ values, and $j$ has $\xi$ values. By use of the inverse of the unitary transformation (9), and of (7), it is clear that the arith- 
metics of $Q\left(\alpha_{1}, \alpha_{2}, \alpha_{3}\right)$ are also the arithmetics of $Q\left(\gamma_{1}, \gamma_{2}, \gamma_{3}\right)$ and of $Q(\alpha, \beta)$. Hence

THEOREM II. The numbers of (28) constitute the bases for the arithmetics of $Q\left(\alpha_{1}, \alpha_{2}, \alpha_{3}\right)$, of $Q\left(\gamma_{1}, \gamma_{2}, \gamma_{3}\right)$, and of $Q(\alpha, \beta)$. There are $2^{k} \xi$ such arithmetics.

The number of arithmetics agrees with that determined by Latimer and Darkow, and the bases were checked by applying the theorem mentioned in footnote 3 . In this theorem the fundamental number $d$ of the algebra is used. By a known result ${ }^{11}$

$$
d= \pm 2^{1-k} B_{1} B_{2} B_{3}
$$

the sign being positive if $\alpha_{1}, \alpha_{2}, \alpha_{3}$ are all of the same sign, and negative otherwise; that is, according as $N(Q)$ (see (3)) is a definite or indefinite form. It is also known that $Q(\alpha, \beta)$ is a division algebra if and only if $d \neq-1 .^{15}$

5. The method of writing down the arithmetics of $Q(\alpha, \beta)$, or of $Q\left(\gamma_{1}, \gamma_{2}, \gamma_{3}\right), \alpha$ and $\beta$ containing no squared prime factors. By use of (7) and (9), and a few new symbols, the arithmetics may readily be written down in terms of the original basis, $\left(I_{0}, I_{1}, I_{2}, I_{1} I_{2}\right)$. Let the following definitions and order of procedure be made:

(a) $(\alpha, \beta)=\gamma_{3}$ (positive or negative), so that $\alpha=-\gamma_{2} \gamma_{3}, \beta=-\gamma_{3} \gamma_{1}$.

(b) $\gamma_{r}=\Gamma_{r} \Delta_{r}$, and

$$
\left(\frac{-\gamma_{s} \gamma_{t}}{\Gamma_{r}}\right)=1, \quad\left(\frac{-\gamma_{s} \gamma_{t}}{\Delta_{r}}\right)=-1,
$$

$\Delta_{r}$ a positive minimum and (rst) a permutation of (123).

(c) Arrange $\gamma_{1}, \gamma_{2}, \gamma_{3}$ (changing signs of all simultaneously if necessary) and determine $\theta$ so that $\gamma_{t} \equiv \theta(\bmod 2 \theta), \gamma_{s} \equiv 1(\bmod 4), \gamma_{r} \equiv \gamma_{s}$ $(\bmod 2(3-\theta))$.

(d) Then determine $m$ so that $\gamma_{r}+\gamma_{s}+m \gamma_{t} \equiv 2-\theta(\bmod 4 \theta)$, $(m-1)(m+1)[(\theta-1) m(m+2)+2-\theta]=0$.

(e) Determine $k$ and $n$ such that $2\left(m^{2}+m+1\right) k=(m+1)(m+2)$, and $k n^{2}-n=0$.

(f) Determine $f_{1}, f_{2}, \cdots, f_{8}$ according to Table I.

(g) Determine the $\xi$ solutions $H_{j}(j=1,2, \cdots, \xi)$ of $\left(\gamma_{1} \gamma_{2}+\gamma_{2} \gamma_{3}\right.$ $\left.+\gamma_{3} \gamma_{1}\right) H_{j}^{2} \equiv-1\left(\bmod \Gamma_{1} \Gamma_{2} \Gamma_{3}\right), \xi$ being the number of prime factors of $\Gamma_{1} \Gamma_{2} \Gamma_{3}$. If any $H_{j}$ is even, replace it by $H_{j} \pm \Gamma_{1} \Gamma_{2} \Gamma_{3}$ to make it odd.

${ }^{15}$ Latimer, C. G., loc. cit., footnote 11 . See $\$ 1$, next to last paragraph, and the corollary to the theorem on page 435 . 
If then any $H_{j}$ be such that $\Gamma_{s} \Delta_{r} H_{j} \not \equiv f_{7}\left(\bmod f_{8}\right)$, replace it by $H_{j} \pm \Gamma_{1} \Gamma_{2} \Gamma_{3}$ and find that $\Gamma_{s} \Delta_{r} H_{j} \equiv f_{7}\left(\bmod f_{8}\right)$.

(h) Then determine $V_{0 j n}, V_{1 j n}, V_{2 j n}, V_{3 j n}$, as the desired bases, for each value of $j$ and $n$, where

$$
\begin{aligned}
& V_{0 j n}=I_{0}, \quad V_{1 j n}=\frac{f_{1} I_{0}+f_{2} I_{r}}{2}, \\
& V_{2 j n}=\frac{f_{3} I_{0}+f_{4} I_{r}}{2}+f_{5} \frac{\gamma_{r} H_{j} I_{r}+I_{s}}{2 \Gamma_{t}} \\
& V_{3 j n}=\frac{I_{0}+f_{6} I_{r}}{2}+\frac{-\gamma_{r} H_{j} I_{r}+\gamma_{s} H_{j} I_{s}+I_{t}}{2 \Gamma_{r} \Gamma_{s}} .
\end{aligned}
$$

(i) Finally, replace $\alpha_{3} I_{3}$ by $I_{1} I_{2}$, giving the final forms.

Hull ${ }^{16}$ has solved the problem of determining a set of bases for all

TABLE I

\begin{tabular}{|l|rrrrrrrrr|}
\hline$\theta$ & 1 & 1 & 1 & 2 & 2 & 2 & 2 & 2 & 2 \\
$m$ & -1 & 1 & 1 & 1 & 1 & 0 & 0 & -1 & -2 \\
$k$ & 0 & 1 & 1 & 1 & 1 & 1 & 1 & 0 & 0 \\
$n$ & 0 & 0 & 1 & 0 & 1 & 0 & 1 & 0 & 0 \\
\hline$f_{1}$ & 0 & 0 & 1 & 0 & 0 & 0 & 0 & 0 & 0 \\
$f_{2}$ & 2 & 2 & 1 & 2 & 2 & 2 & 2 & 2 & 2 \\
$f_{3}$ & 0 & 1 & 0 & 1 & 1 & 0 & 0 & 0 & 0 \\
$f_{4}$ & 0 & 1 & 0 & 0 & 1 & 0 & 1 & 0 & 0 \\
$f_{5}$ & 2 & 1 & 2 & 1 & 1 & 1 & 1 & 2 & 2 \\
$f_{6}$ & 0 & 0 & 0 & 1 & 1 & 0 & 0 & 1 & 0 \\
$f_{7}$ & 3 & -1 & -1 & -1 & -1 & 1 & 1 & 3 & 5 \\
$f_{8}$ & 2 & 2 & 2 & 4 & 4 & 4 & 4 & 2 & 2 \\
\hline
\end{tabular}

the arithmetics of division algebras, by showing that every arithmetic may be obtained from some one of an infinite number of canonical generations of the algebra (merely a non-unitary transformation of units carrying $\alpha$ and $\beta$ into $\alpha^{\prime}$ and $\beta^{\prime}$ having special properties). Latimer has obtained similar results without the restriction that the algebras be division algebras. ${ }^{3}$ However, the method of determining which canonical generations will give the arithmetics for a given un-

${ }^{16}$ Hull, Ralph, The maximal orders of generalized quaternion division algebras, Transactions of this Society, vol. 40 (1936), pp. 1-11. 
altered $\alpha$ and $\beta$ is not stated in either paper. Hull showed that the canonical forms determined by Albert ${ }^{8}$ are special cases of his canonical generations.

One problem remains to be solved, namely a method of writing down the arithmetics of $Q(\alpha, \beta)$ when $\alpha$ and $\beta$ contain squared prime factors.

UNIVERSITY OF ILLINOIS 